\title{
Orbital involvement in sickle cell disease: a report of five cases and review literature
}

\begin{abstract}
Purpose To present five cases of orbital infarction in sickle cell disease and review relevant literature.

Method We reviewed the hospital records of 5 patients with sickle cell disease who developed a periorbital swelling during a vaso-occlusive crisis and were managed at our hospital between April 1992 and June 2000. Results The 5 patients ( 4 with homozygous sickle cell disease and 1 with sickle cell$\beta$-thalassaemia disease) were aged 6-15 years with a history of multiple admissions for vaso-occlusive crises. The periorbital swelling spread to the orbit in 4 cases and resulted in proptosis ( 2 cases), restriction of ocular motility and visual impairment. In all 4 cases, computed tomography and/or magnetic resonance imaging of the orbits showed a mass adjacent to the orbital wall. In 2 cases the mass was identified as a haematoma. Orbital wall infarction was demonstrated in $\mathbf{3}$ cases by bone/bone marrow scintigraphy. Epidural haematomas were detected by computed tomography in one case. All patients received intravenous fluids, analgesics, broad spectrum antibiotics and steroids, as well as simple or exchange transfusion, and responded well to medical management.

Conclusions Infarction of orbital bones during vaso-occlusive crises in sickle cell disease presents acutely with a rapidly progressive periorbital swelling. Haematomas frequently complicate the condition and, along with the inflammatory swelling, may lead to orbital compression syndrome. The condition is therefore sight-threatening, and necessitates prompt diagnosis and appropriate management for resolution without adverse sequelae. Imaging techniques are invaluable in the evaluation of patients. The majority of cases resolve with conservative treatment that includes steps to combat the vaso-occlusive crisis and use of systemic steroids under antibiotic cover.
\end{abstract}

Key words Management, Orbital compression syndrome, Orbital haemorrhage, Orbital infarction, Sickle cell disease
ANURADHA GANESH, RANJAN R. WILLIAM, SANDIP MITRA, SUJATHA YANAMADALA, SAMIR S. HUSSEIN, SALAM AL-KINDI, MATHEW ZAKARIAH, ZAKIA AL-LAMKI, HUXLEY KNOX-MACAULAY
Ocular involvement in sickle cell disease (SCD) is characterised by retinopathy, anterior segment ischaemia, glaucoma and angioid streaks, and has been well documented. ${ }^{1}$ In comparison, orbital involvement in SCD with infarction of orbital bones is less well recognised. ${ }^{2}$ However, awareness of this condition is necessary, as inappropriate management can rapidly lead to visual impairment. We present 5 cases of orbital infarction in SCD managed at our hospital between April 1992 and June 2000, and discuss the clinical features, diagnostic investigation and management. To our knowledge, this is the largest series of this entity in the ophthalmic literature.

\section{Case reports}

\section{Case 1}

A 13-year-old boy with homozygous sickle cell (HbSS) disease presented with low-grade fever and pain in the back and forearm, and was admitted with a diagnosis of vaso-occlusive crisis (VOC). Treatment with intravenous fluids and morphine was commenced. The next day, he developed a painful right eyelid and facial swelling. Intravenous cefuroxime was administered and 1 unit of packed red blood cells was transfused, but the swelling increased and the patient complained of increasing pain and decreasing vision on the right side. Ophthalmic examination revealed bilateral eyelid oedema with minimal erythema, worse on the right side, restriction of ocular motility in abduction, and conjunctival hyperaemia with chemosis in the right eye (Fig. 1). Visual acuity was $6 / 18$ in the right eye and 6/6 in the left. Pupillary reflexes and fundi were normal.

Computed tomography (CT) of the orbits showed bilateral, periorbital soft tissue swelling. An extraconal mass measuring 40 Hounsfield units (HU) was detected adjacent to the lateral orbital wall on the right side. Blood culture was negative.

Intravenous dexamethasone was administered and resulted in a rapid resolution of eyelid oedema, restriction of motility and 


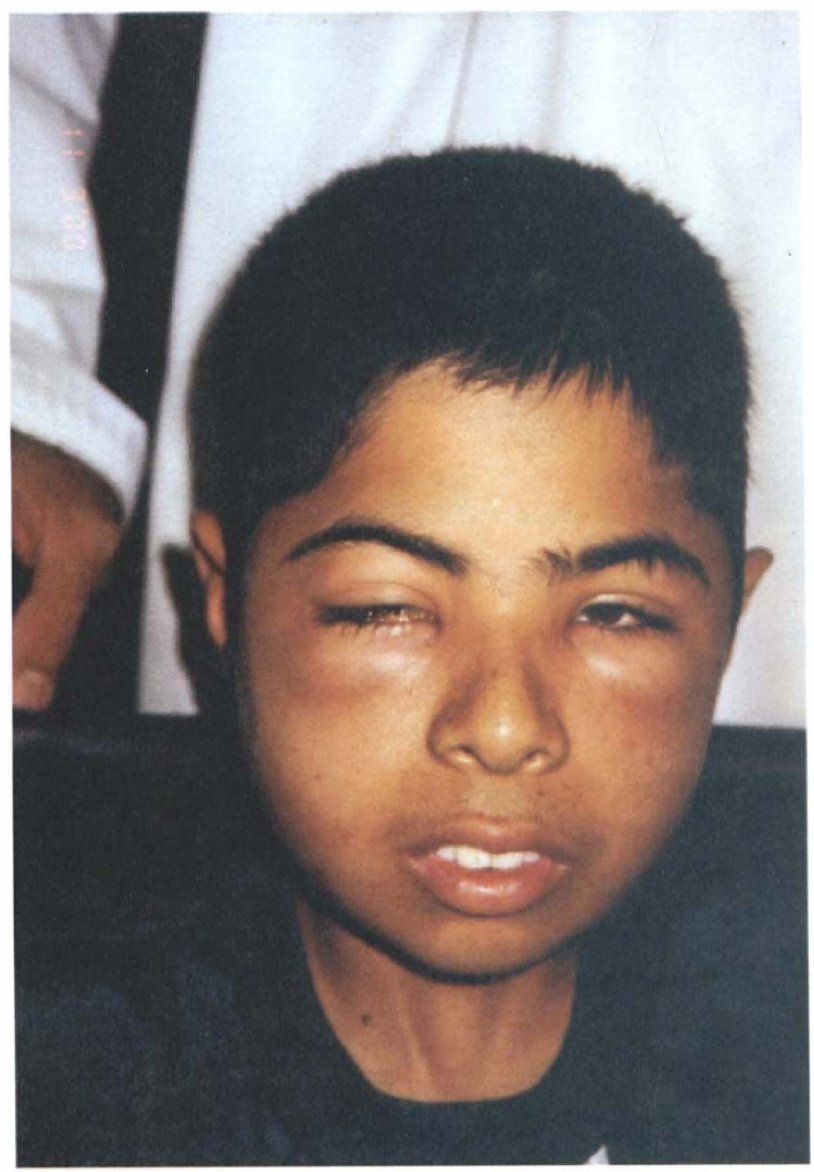

Fig. 1. Case 1. Face photograph showing bilateral eyelid oedema with minimal erythema. Note the conjunctival hyperaemia and chemosis in the right eye, which is more severely affected.

conjunctival chemosis. Visual acuity improved in the next 2 days to $6 / 6$ and the patient was discharged on oral antibiotics and folic acid.

\section{Case 2}

An 11-year-old boy with HbSS disease was admitted with a diagnosis of VOC after he developed fever, painful swelling of the right eye and pain in the limbs. Ophthalmic evaluation showed visual acuity of $6 / 60$ in the right eye and 6/6 in the left, and right-sided lid oedema, proptosis and conjunctival hyperaemia with chemosis (Fig. 2a). Ocular motility was restricted in all directions and the cornea was hypoesthetic with a relative afferent pupillary defect in the right eye. Intraocular pressure and the fundus were normal. The patient also manifested two subcutaneous swellings in the parieto-occipital region of the scalp. These were soft, non-tender and fluctuant, with the larger of the swellings being $2 \times 1.5 \mathrm{~cm}$ in size.

$\mathrm{CT}$ of the orbits showed right periorbital swelling. An extraconal mass with an attenuation value consistent with that of a haematoma (50-60 HU) was seen adjacent to the lateral orbital wall on the right side. The retrobulbar space and extraocular muscles were unaffected (Fig. 2b). CT of the brain showed bi-frontal epidural haematomas (Fig. 2c). Bone scintigraphy with
${ }^{99 m}$ Tc-methylene diphosphonate in the static phase revealed multiple photopenic areas in the skull compatible with infarction (Fig. 2d).

Fine needle aspiration of a scalp swelling was performed and $2 \mathrm{ml}$ of sero-sanguineous fluid was aspirated. Microscopic examination showed numerous granulocytes and degenerated cells. Culture of the aspirate and blood yielded no organisms. Investigations for bleeding disorders (complete blood count, bleeding time, prothrombin time, activated partial thromboplastin time and factor assays) revealed no abnormality.

The patient was treated with intravenous fluids, blood transfusion, analgesics and broad spectrum antibiotics. A reduction in the periocular and orbital swelling was observed within $24 \mathrm{~h}$, and both eye and scalp swellings completely resolved within 2 weeks.

\section{Case 3}

A 6-year-old boy with sickle cell- $\beta$-thalassaemia (HbS $\beta$ Thal) disease and glucose-6-phosphatedehydrogenase deficiency developed bilateral lid swellings with low-grade fever and pain in the back and lower limbs, and was admitted with a diagnosis of VOC. Intravenous fluids, analgesics and ceftriaxone were commenced. Ophthalmic examination revealed bilateral, extensive periorbital oedema and proptosis of 21 and $25 \mathrm{~mm}$ in the right and left eye respectively (Fig. 3a). Visual acuity was $6 / 6$ in the right eye and 6/18 in the left, with bilateral restriction of ocular motility in horizontal and up-gaze. Pupillary reactions, intraocular pressure and fundi were normal in both eyes.

CT of the orbits showed periocular swelling and high attenuating (50-60 HU) extraconal collections in the superolateral region of both orbits (Fig. 3b). Magnetic resonance imaging (MRI) of the orbits performed on the same day with a $1.5 \mathrm{~T}$ unit disclosed bilateral extensive inflammatory changes and subperiosteal fluid collections in the superolateral orbital regions. The collections were iso- to hypo-intensive on T1-weighted images and hypo-intense on T2-weighted images, and were therefore identified as haematomas of recent origin (Fig. 3c). Fat-saturation T2-weighted coronal images revealed normal optic nerves. The sphenoid wing and frontal bones showed evidence of marrow hyperplasia and bone infarction with heterogeneous signal intensities on T1- and T2-weighted images. Bone marrow scintigraphy performed the next day with ${ }^{99 \mathrm{~m}} \mathrm{Tc}-\mathrm{Nanacoll}$

(Nycom-Amersham, UK) revealed multiple low-uptake regions in the skull, predominantly in the left frontal and sphenoid wing region, confirming orbital wall infarction (Fig. 3d). Investigations for bleeding disorders revealed no abnormalities and blood culture was negative.

Intravenous dexamethasone was administered and exchange transfusion was carried out. The response to this treatment was dramatic, with improvement in vision to $6 / 6$ in the left eye, reduction in lid oedema and proptosis, and restoration of extraocular motility over the next 2 days. The patient was discharged with mild residual proptosis $(23 \mathrm{~mm})$ of the left eye (Fig. 3e). 


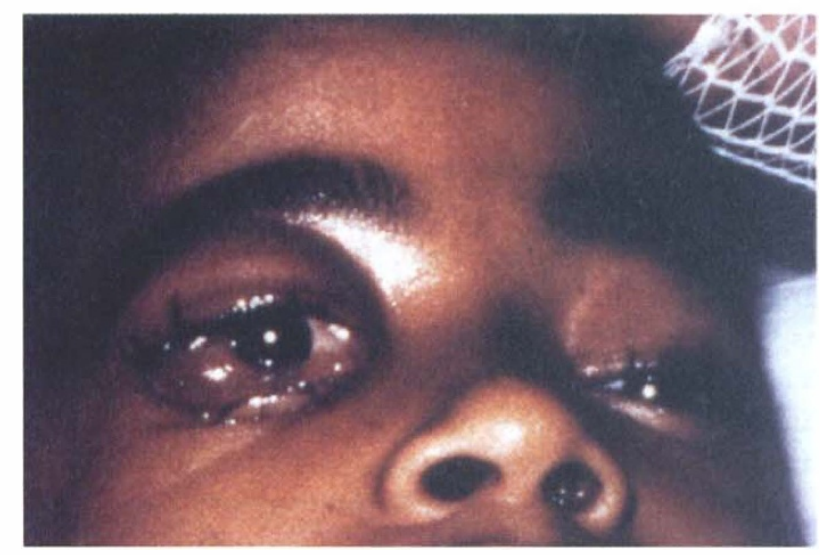

(a)

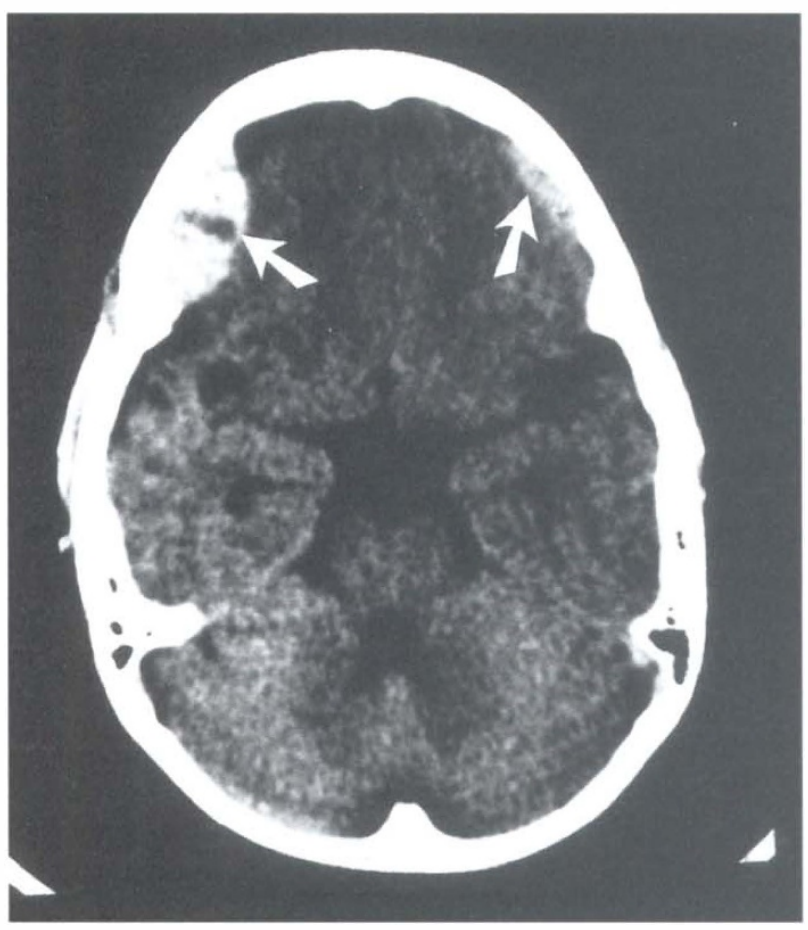

(c)

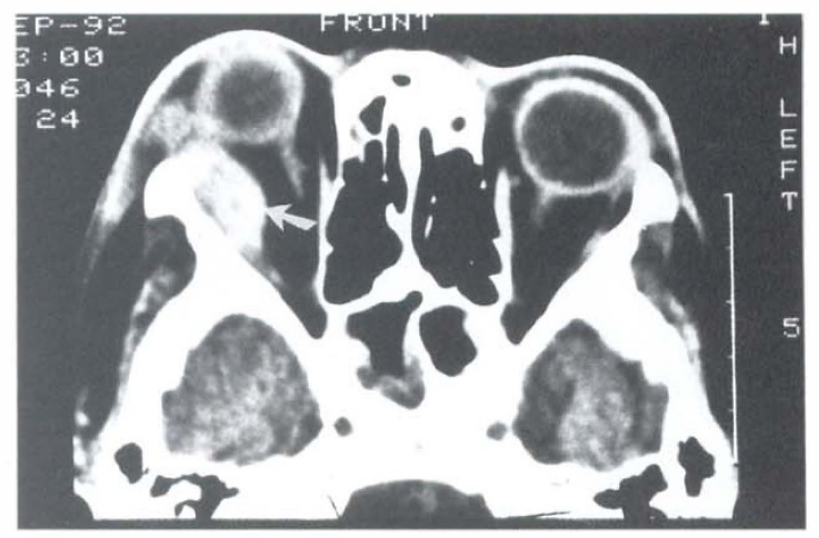

(b)

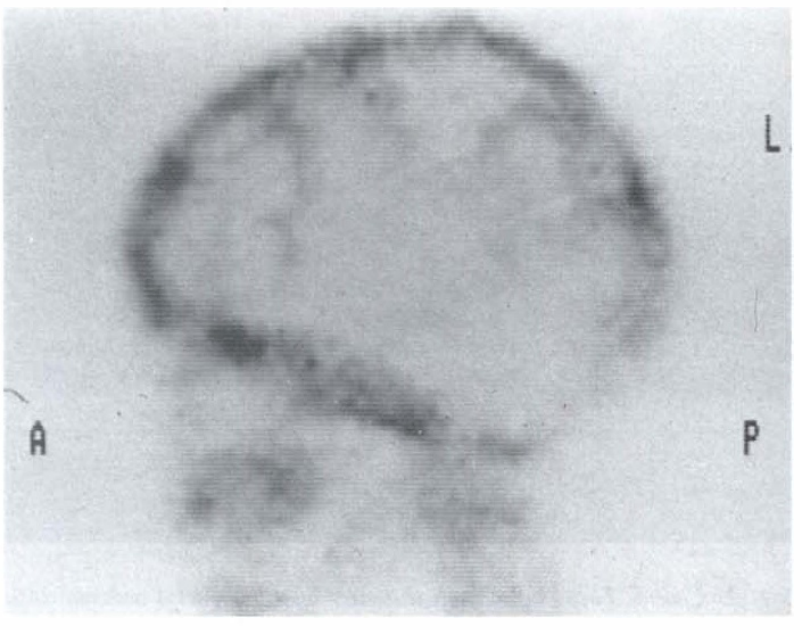

(d)

Fig. 2. Case 2. (a) Eyelid oedema, proptosis and conjunctival hyperaemia with chemosis in the right eye. (b) Axial CT image of the orbits showing right periorbital swelling and an extraconal mass (>50 HU) adjacent to the lateral wall of the right orbit (arrow). (c) Axial CT image of the brain showing bilateral epidural haematomas in the frontal region, right more than left (arrows). (d) ${ }^{99 m}$ Tc bone scintigraphy: lateral view showing decreased uptake in multiple areas in the skull.

\section{Case 4}

A 10-year-old boy with HbSS disease and history of a cerebrovascular accident (left hemiplegia) 2 years previously, was admitted for management of VOC.

Treatment was commenced with intravenous fluids and analgesics. On the second day he developed pain around the right eye with swelling of the eyelids and right side of the face. On examination, vision was $6 / 6$ in both eyes. There was marked right-sided periorbital oedema and conjunctival chemosis, with restriction of ocular motility in abduction. The pupil was normal in size and reacted briskly to light, and the fundus was normal. Intravenous ceftriaxone was commenced.

CT of the orbits showed periocular swelling and an extraconal soft tissue lesion $(40 \mathrm{HU})$ in the right orbit.
Bone scintigraphy was normal in this patient and blood culture was negative.

Exchange transfusion was carried out the next day and intravenous dexamethasone was administered. The ocular swelling resolved completely over the next 3 days and the patient was discharged.

\section{Case 5}

A 15-year-old boy with HbSS disease was admitted with complaints of pain in the back, abdomen and lower limbs. Treatment with intravenous fluids and analgesics was initiated. Overnight, he developed a left periocular swelling. This was mildly painful, erythematous and 


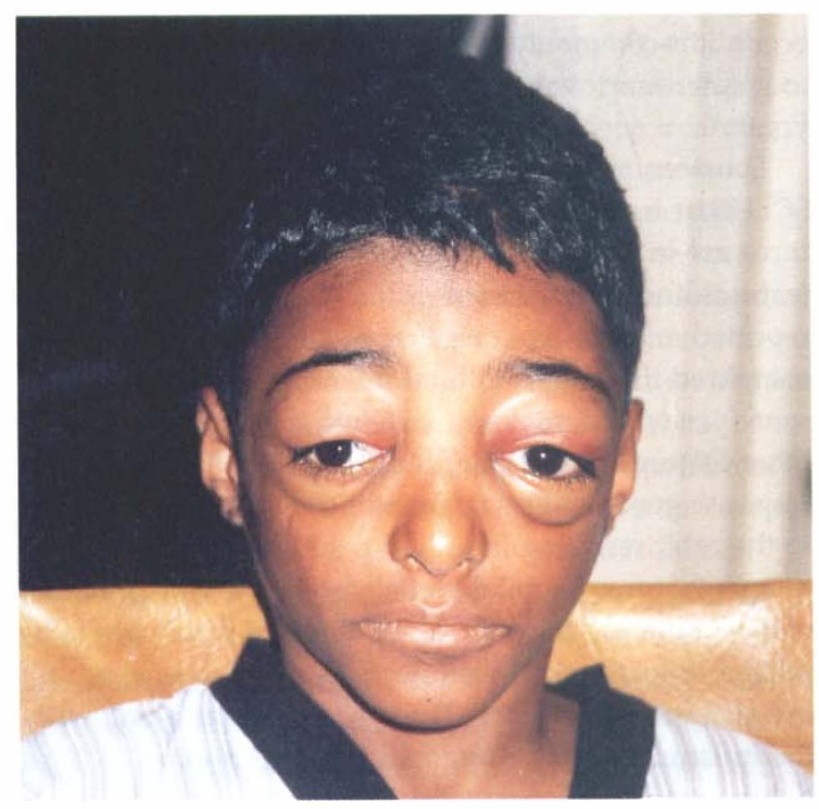

(a)

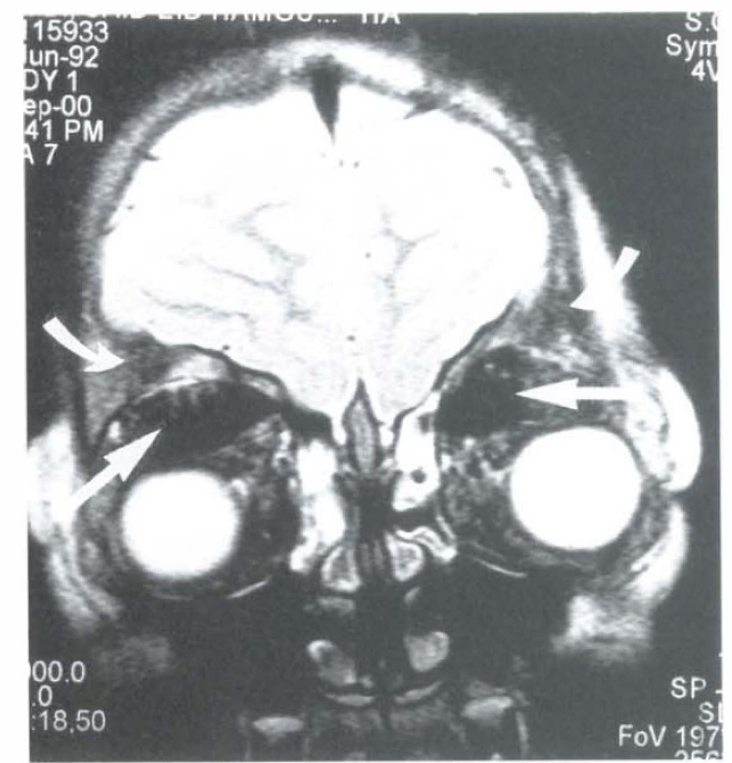

(c)

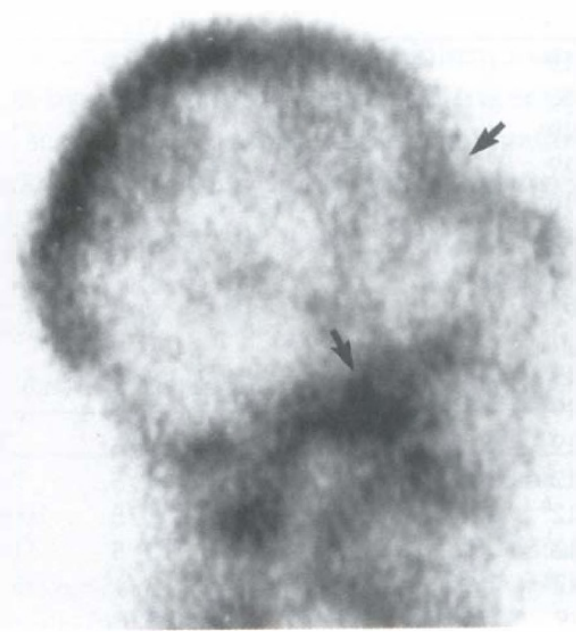

(d)

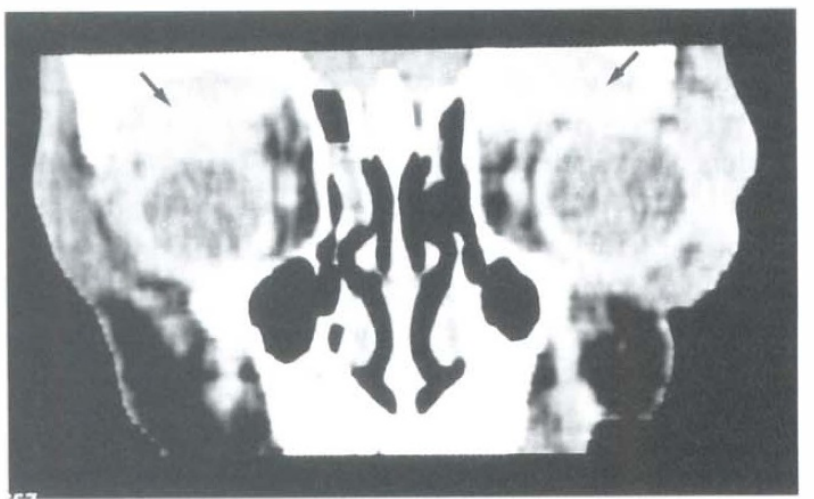

(b)

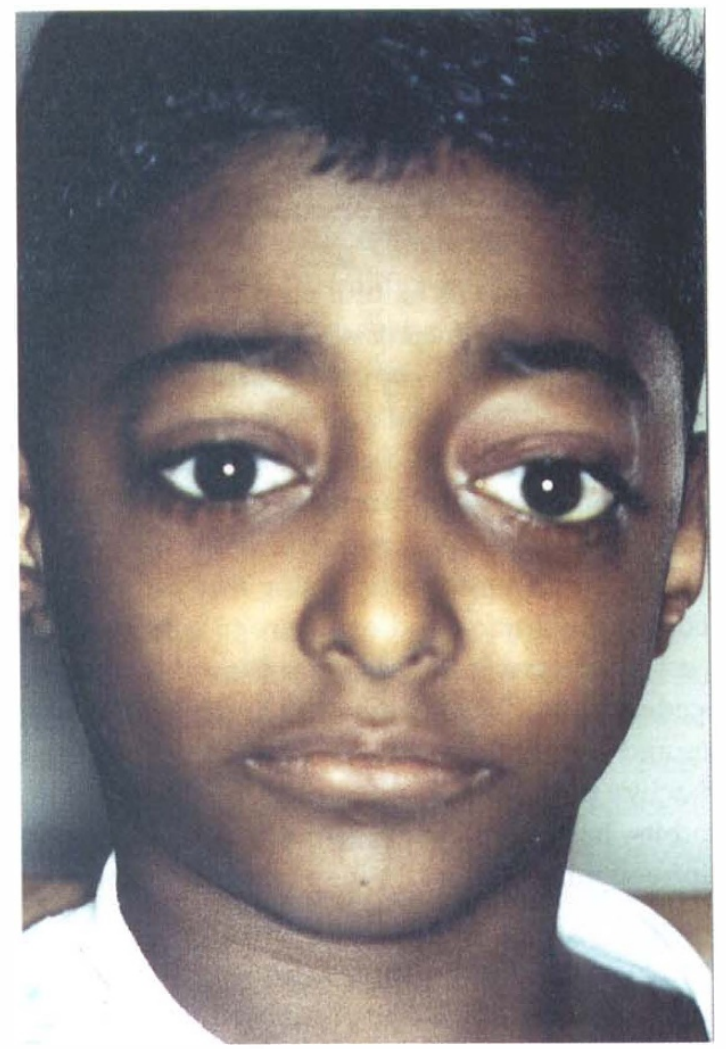

(e)

Fig. 3. Case 3. (a) Face photograph showing bilateral, extensive and erythematous eyelid swellings with proptosis. (b) Coronal reconstructed CT image of the orbits showing bilateral extraconal lesions (> $50 \mathrm{HU}$ ) adjacent to superior orbital walls (arrows). (c) T2-weighted, fat-saturation, coronal MR image of the orbits showing bilateral low signal, subperiosteal fluid collections in the superolateral orbital regions (straight arrows). Note also the heterogeneous signals from the marrow of the sphenoid bones (curved arrows). (d) ${ }^{99 m}$ Tc bone marrow scintigraphy: lateral view showing multiple low-uptake regions in the skull, predominantly in the frontal and sphenoid wing region (arrows). (e) Face photograph of the patient prior to discharge showing mild residual proptosis of the left eye. 
tender. There was no evidence of any ocular adnexal infection. The rest of the ocular examination was normal. Intravenous cefuroxime was administered.

Bone scintography revealed a photopenic area in the left orbital wall. An immediate CT scan could not be performed; however, 4 days later, CT of the orbits and brain showed no abnormality.

The patient responded well to conservative treatment.

\section{Discussion}

Sickle cell disease (SCD) is a hereditary

haemoglobinopathy wherein a single amino acid substitution in the beta chain of the globin molecule causes red blood cells to become sickle-shaped and rigid under conditions of hypoxia and acidosis. These cells then tend to block capillaries and cause infarctions. The Sultanate of Oman has a high prevalence of $\mathrm{SCD}^{3}$ and the condition accounts for considerable morbidity in the region.

Recurrent attacks of painful vaso-occlusive crises (VOC) resulting from necrosis of bone marrow following obstruction to its blood supply by sickled cells, account for majority of acute hospital admissions in children with SCD. ${ }^{4}$ The pain is believed to be consequent upon the increased intramedullary pressure generated by the inflammatory response to avascular bone marrow. ${ }^{5}$ Although a number of precipitating factors including infection, dehydration and exposure to cold have been suggested, in most instances there is no discernible antecedent cause. VOC commonly involves the long bones and vertebrae, but can in principle affect all bones with active marrow. Facial bones, particularly those of the orbits, have very little marrow space and therefore are infrequently affected. ${ }^{5}$ When orbital infarction does occur, it is commonly encountered in young patients due to greater marrow space in the orbital bones during youth. ${ }^{6}$

Fourteen reports of 19 cases of orbital infarction in SCD exist in the English literature, $;^{2,7-19}$ of these only three are in the ophthalmic literature. The clinical features and management of the 19 cases previously reported and the five cases reported in this paper are compared in Table 1. Orbital infarction presents with acute periorbital pain and swelling in association with other features of the painful crisis. The inflammatory response generated by infarcted bone can rapidly spread to the orbit resulting in orbital pain and proptosis. A unique feature of orbital bone infarction is the formation of haematomas, ${ }^{2}$ the occurrence of which is thought to be related to local vessel wall necrosis and subsequent extravasation of blood. ${ }^{17}$ These haematomas along with the inflammatory swelling may lead to proptosis, ophthalmoplegia, conjunctival chemosis, corneal hypoesthesia and optic nerve dysfunction (orbital compression syndrome). Four of our patients developed signs of orbital compression syndrome consequent upon the presence of an orbital haematoma (cases 2 and 3) and reactive inflammatory swelling adjacent to infarcted bone (cases 1 and 4). The rapid resolution of symptoms and signs upon administration of systemic steroids strongly suggests a predominant inflammatory component consequent upon the orbital bone infarction due to sicklaemic vaso-occlusion, in the pathogenesis of orbital compression syndrome.

One of the patients (case 2) in this series also developed epidural haematomas. These are almost always of post-traumatic origin, but occasionally may be related to rupture of a dural vascular malformation or a bleeding disorder. Epidural haematomas have been

Table 1. Comparison of data from published $(A)$ and present $(B)$ reports of orbital infarction in sickle cell disease

\begin{tabular}{|c|c|c|}
\hline \multirow[b]{2}{*}{ Clinical features } & \multicolumn{2}{|c|}{ No. of patients } \\
\hline & $\mathrm{A}(n=19)^{a}$ & $\mathrm{~B}(n=5)^{a}$ \\
\hline Age $<10$ years & $19 / 19$ & $5 / 5$ \\
\hline Sex: $M / F$ & $11 / 8$ & $5 / 0$ \\
\hline History of multiple VOCs & $19 / 19$ & $5 / 5$ \\
\hline Presenting symptoms - pain, lid oedema & $19 / 19$ & $5 / 5$ \\
\hline Fever & $15 / 16^{a}$ & $5 / 5$ \\
\hline Pain crisis & $11 / 16^{a}$ & $5 / 5$ \\
\hline Orbital signs (proptosis/visual loss/ophthalmoplegia) & $17 / 19$ & $4 / 5$ \\
\hline Unilateral/bilateral & $11 / 6$ & $3 / 1$ \\
\hline \multicolumn{3}{|l|}{ Haemoglobinopathy } \\
\hline Homozygous sickle cell disease & $17 / 19$ & $4 / 5$ \\
\hline Sickle cell-haemoglobin C disease & $1 / 19$ & $0 / 5$ \\
\hline Sickle cell- $\beta$-thalassaemia disease & $1 / 19$ & $1 / 5$ \\
\hline Orbit haematoma (CT) & $7 / 12^{b}$ & $2 / 5$ \\
\hline Orbit abscess (CT) & $2 / 12^{b}$ & $0 / 5$ \\
\hline Intracranial haematoma $(\mathrm{CT})$ & $3 / 12^{b}$ & $1 / 5$ \\
\hline Bone infarction (bone/bone marrow scan) & $12 / 12^{b}$ & $3 / 4^{b}$ \\
\hline Conservative management and recovery & $14 / 19$ & $5 / 5$ \\
\hline Surgical management and recovery & $5 / 19$ & $0 / 5$ \\
\hline Permanent visual loss & $1 / 19$ & $0 / 5$ \\
\hline
\end{tabular}

VOCs, vaso-occlusive crises.

an, total number of patients.

${ }^{b}$ Not reported/performed in remaining cases. 
observed in $\mathrm{SCD},{ }^{20,21}$ at times accompanying orbital infarction, ${ }^{13,14,17}$ and are thought to result from diploic venous thrombosis subsequent to skull bone infarction.

Patients with SCD are immunocompromised and prone to serve infections, ${ }^{5}$ particularly osteomyelitis, ${ }^{22}$ which shares many clinical features with acute bone infarction and presents a diagnostic dilemma. Indeed orbital abscess formation at the site of ishaemic and necrotic bone has been reported; ${ }^{16,19}$ however, we did not come across any reports of osteomyelitis of orbital bones complicating VOC in SCD. The presence of persistent pain not resolving with the usual supportive therapy for sickle cell crisis, high-grade fever with chills and an erythematous and exquisitely tender swelling should alert the clinician to the possibility of superadded infection. Investigations for sepsis (blood culture, culture of bone aspirate/periosteal effusion) should confirm diagnosis. None of the cases in this series manifested features suggestive of infection; moreover microbiological studies were negative in all of them.

Various imaging modalities have been employed in evaluating sickle cell patients with bone infarctions. ${ }^{18,23-28} \mathrm{CT}$ and MRI have an important role in narrowing the differential diagnosis in cases with orbital involvement (Table 2). However, CT fails to show any bone abnormality and findings in bone infarction are limited to soft tissue swelling and fluid collections. ${ }^{18} \mathrm{CT}$ can identify a mass as a collection of blood when its attenuation value is over $50 \mathrm{HU}$, as in cases 2 and 3. MRI is a more sensitive and specific imaging technique that can reliably distinguish between vascularised inflammatory tissue and afluid collection. It can further characterise the latter as an abscess or haematoma, ${ }^{18,23}$ as was seen in case 3. MRI has the added advantage of being capable of delineating ischaemic changes in the marrow space. ${ }^{24}$ However, studies evaluating the diagnostic utility of contrast enhanced MRI in differentiating the osseous changes of acute bone infarction from osteomyelitis have yielded conflicting results. $^{23,25}$

Bone marrow scintigraphy can confirm marrow infarction by demonstrating decreased tracer uptake. Marrow scan can also, especially in combination with ${ }^{67} \mathrm{Ga}$ citrate scintigraphy, differentiate osteomyelitis from infarction. ${ }^{26}$

Table 2. Causes of acute periorbital/orbital swelling

\begin{tabular}{ll}
\hline Infective & Non-infective \\
\hline Cellulitis $^{a}$ & Rhabdomyosarcoma \\
Preseptal & Neuroblastoma \\
Orbital & Acute myeloid leukaemia \\
Orbital abscess & Other metastatic lesions \\
Osteomyelitis (orbital bones) & Pseudotumour \\
& Lymphangioma/dermoid cyst \\
& Capillary haemangioma \\
& Traumatic haematoma \\
\hline
\end{tabular}

${ }^{a}$ Usually secondary to upper respiratory tract infection, trauma or local sepsis in eyelid, lacrimal sac or conjunctiva.
The results of bone scintigraphy are dependent on the timing of the study. There is a reduced tracer uptake during the acute stage (first $48 \mathrm{~h}$ ) indicating marrow death and reduced vascularity. Later, despite persistence of marrow inactivity, bone scans may demonstrate an increased uptake reflecting the increased vascularity of the healing phase. ${ }^{27}$ This may explain the negative results of bone scintigraphy in case 4 .

Ultrasonography is a simple and effective imaging tool that can differentiate bone infection from infarction in SCD. ${ }^{28}$ However, its efficacy has not been evaluated in sickle cell patients with orbital involvement.

All the patients reported in this series presented with moderate to severe periorbital/orbital swellings during a VOC. CT and bone marrow scintigraphy were performed in all of them to establish the diagnosis of orbital wall infarction, and to evaluate the extent of soft tissue swelling. MRI was performed in only one patient (case 3), in whom the findings on CT suggested the presence of orbital haematomas. MRI confirmed the nature of the fluid collections as haematomas and also delineated the posterior extent of the space-occupying lesions.

It is worth remembering that crises are repetitive events; thus, the bone marrow of sickle cell patients is likely to show effects of past as well as present VOCs. Additionally, there is considerable overlap of findings in bone infarction and osteomyelitis. This often necessitates a multimodality approach to confirm the diagnosis. ${ }^{23,28}$

Most cases of orbital infarction in SCD resolve with conservative management that includes treatment of the VOC with intravenous fluids and analgesics. ${ }^{29}$ Blood transfusions or exchange transfusions that reduce the level of sickle cells in the circulation are not routinely considered in the management of VOC, but are performed in special situations such as crises that fail to resolve despite treatment, and in patients incapacitated by frequent recurrences. Empiric use of broad spectrum intravenous antibiotics is advisable because sickle cell patients are susceptible to infection and it is often difficult to clinically differentiate infarction from infection. However, as acute bone infarction is at least 50 times more common than bacterial osteomyelitis, it is important that patients not be overtreated. ${ }^{30}$

In cases that do not show signs of resolution with the above therapeutic measures, administration of intravenous steroids under antibiotic cover is recommended. This is particularly important as the orbit is a cavity with limited space and inflammatory swellings that are not controlled promptly may compromise important structures. Signs of optic nerve dysfunction with radiological evidence of a haematoma mandates surgical evacuation of the haematoma. While 14 of the 19 patients reported previously in the literature recovered completely with medical therapy, 5 underwent frontal sinus trephination or orbitotomy for evacuation of a haematoma. All the 5 patients reported in our study responded well to conservative treatment. 


\section{Summary}

This paper describes the clinical features and management of orbital infarction in 5 patients with SCD. These cases highlight the importance of close monitoring of physical signs to detect features of orbital compression syndrome and infection, the usefulness of imaging techniques in the evaluation of patients, and the satisfactory response to conservative management. A heightened suspicion of orbital infarction in sickle cell patients with ocular symptoms is essential to allow prompt diagnosis and treatment.

\section{References}

1. Nagpal KC, Goldberg MF, Rabb MF. Ocular manifestations of sickle hemoglobinopathies. Surv Ophthalmol 1977;21:391-411.

2. Curran EL, Fleming JC, Rice K, Wang WC. Orbital compression syndrome in sickle cell disease. Ophthalmology 1997;104:1610-5.

3. Al-Riyami A. Sickle Cell. In: Al Riyami, ed. National genetic blood disorders survey. Sultanate of Oman: Ministry of Health, 1995;19-24.

4. Gill FM, Sleeper LA, Weiner SJ, Brown AK, Bellevue R, Grover $\mathrm{R}$, et al. Clinical events in the first decade in a cohort of infants with sickle cell disease. Blood 1995;86:776-8.

5. Serjeant GR. Sickle cell disease, 2nd ed. Oxford: Oxford University Press, 1992.

6. Stool SE, Marasovich WA. Postnatal craniofacial growth and development. In: Pediatric otolaryngology, 2nd ed. Philadelphia: WB Saunders, 1990.

7. Al-Rashid RA. Orbital apex syndrome secondary to sickle cell anaemia. J Pediatr 1979;95:426-7.

8. Blank JP, Gill FM. Orbital infarction in sickle cell disease. Pediatrics 1981;67:879-81.

9. Seeler RA. Exophthalmos in haemoglobin SC disease. J Pediatr 1983;102:90-1.

10. Garty I, Koren A, Garzozi H. Frontal and orbital bone infarctions causing periorbital swelling in patients with sickle cell anemia. Arch Ophthalmol 1984;102:1486-8.

11. Wolff MH, Sty JR. Orbital infarction in sickle cell disease. Pediatr Radiol 1985;15:50-2.

12. Ozsoylu S, Jama H, Erturk G, Tokatli A. Orbital apex syndrome due to sickle cell anaemia. Pediatr Hematol Oncol 1986;3:183-5.

13. Seibert RW, Seibert JJ, Frazier EA. Infarction of the orbit and paranasal sinuses in sickle cell disease. South Med J 1987;80:1569-71.

14. Mallouh AA, Young M, Hamdan J, Salamah MM. Proptosis, skull infarction, and retroorbital and epidural hematomas in a child with sickle cell disease. Clin Pediatr 1987;26:536-8.
15. Royal JE, Harris VJ, Sansi PK. Facial bone infarcts in sickle cell syndromes. Radiology 1988;169:529-31.

16. Sidman JD, Brownlee RE, Smith WC, Fry TL. Orbital complications of sickle cell disease. Int J Pediatr Otorhinolaryngol 1990;19:181-4.

17. Karacostas D, Artemis N, Papadopoulu M, Christakis J. Case report: epidural and bilateral retroorbital hematomas complicating sickle cell anaemia. Am J Med Sci 1991;302:107-9.

18. Rebsamen SL, Bilaniuk LT, Granet D, Foster J, Low J, Heyman S, Katowitz J. Orbital wall infarction in sickle cell disease: MR evaluation. AJNR 1993;14:777-9.

19. Nath J, Jin DK, Rahman R, Ballas SK, Batuman OA. Successful medical management of orbital abscess in a patient with sickle cell anaemia. Ophthalmic Surg Lasers 1998;29:860-3.

20. Resar LM, Oliva MM, Casella JF. Skull infarction and epidural haematomas in a patient with sickle-cell anaemia. J Pediatr Hematol Oncol 1996;18:413-5.

21. Cabon I, Hladky JP, Lambilliotte A, Cotton A, Hellemmes P. Uncommon etiology of extradural hematoma. Neurochirurgie 1997;43:173-6.

22. Overturf GD. Infections and immunisations of children with sickle cell disease. Adv Pediatr Infect Dis 1999;14:191-218.

23. Bonnerot, Sebag G, deMontalembert M, Wioland M, Glorion C, Girot R, Lallemand D. Gadolinium-DOTA enhanced MRI of painful osseous crises in children with sickle cell anaemia. Pediatr Radiol 1994;24:92-5.

24. Mankad VN, Williams JP, Harpen MD. Magnetic resonance imaging of the bone marrow in sickle cell disease: clinical hematologic and pathologic correlations. Blood 1990;75:274-83.

25. Umans H, Haramati N, Flusser G. The diagnostic role of gadolinium enhanced MRI in distinguishing between acute medullary bone infarct and osteomyelitis. Magn Reson Imaging 2000;18:255-62.

26. Khan CE Jr, Ryan JW, Hatfield MK. Combined bone marrow and gallium imaging: differentiation of osteomyelitis and infarction in sickle haemoglobinopathy. Clin Nucl Med 1988;13:443-9.

27. Conolly LP, Treves ST. Osteomyelitis complicating other diseases: sickle cell anaemia. In: Paediatric skeletal scintigraphy. New York: Springer, 1988:76.

28. William RR, Hussein SS, Jeans WD, Wali YA, Lamki ZA. A prospective study of soft-tissue ultrasonography in sickle-cell disease patients with suspected osteomyelitis. Clin Radiol 2000;55:307-10.

29. Davies SC. The vaso-occlusive crisis of sickle cell disease. BMJ 1991;302:1551-2.

30. Buchanan GR. Infection. In: Embury SH, Hebbel RP, Mohandas N, Steinberg MH, eds. Sickle cell disease: basic principles and clinical practice. New York: Raven Press, 1994:567-87. 